\title{
Advancing from rules of thumb: quantifying the effects of small density changes in mass transport to electrodes. Understanding natural convection
}

\author{
Kamonwad Ngamchuea, Shaltiel Eloul, Kristina Tschulik*, Richard G. Compton*
}

*corresponding authors: Kristina Tschulik and Richard G. Compton, Department of Chemistry, Physical \& Theoretical Chemistry Laboratory, University of Oxford, South Parks Road, Oxford, OX1 3QZ, United Kingdom

Email: tschulik.kristina@gmail,com, richard.compton@chem.ox.ac.uk. Tel: +44(0)1865275 957 Fax: +44(0)1865275410

\section{Key Words:}

Numerical simulation, convection, diffusion, amperometry, edge effect, density gradient

\begin{abstract}
Understanding mass transport is pre-requisite to all quantitative analysis of electrochemical experiments. While the contribution of diffusion is well-understood, the influence of density gradient driven natural convection on the mass transport in electrochemical systems is not. To date it has been assumed to be relevant only for high concentrations of redox-active species and at long experimental timescales. If unjustified, this assumption risks misinterpretation of analytical data obtained from scanning electrochemical microscopy (SECM), generatorcollector experiments as well as analytical sensors utilising macroelectrodes / microelectrode arrays and it also affects the results expected from electrodeposition. Based on numerical
\end{abstract}


simulation, herein it is demonstrated that even at sub $10 \mathrm{mM}$ concentrations and short experimental times of tens of seconds, density gradient driven natural convection significantly affects mass transport. This is evident by in depth numerical simulation for the oxidation of hexacyanoferrate (II) at various electrode sizes and electrode orientations. In each case, the induced convection and its influence on the diffusion layer established near the electrode are illustrated by maps of the velocity fields and concentration distributions evolving with time. The effects of natural convection on mass transport and chronoamperometric currents are thus quantified and discussed for the different cases studied.

\section{Introduction}

Electrochemistry is key to a number of powerful modern analytical tools and devices. ${ }^{1-11}$ To quantitatively analyse the data obtained, however, both the electrode kinetics and solution phase mass transport governing the system have to be understood. The first can be eliminated by choosing a suitable electrochemical tool and parameter set, such as chronoamperometry at a sufficiently large overpotential. The latter, by contrast, is an inherent feature of any electrochemical reaction. Due to the consumption of reactants and formation of products at the electrode, it has to be considered in all cases. The processes governing mass transport in electrochemical systems are diffusion, migration and convection. Any electrochemical reaction gives rise to diffusion due to the formation of concentration gradients of reactants and products at the electrode. This aspect is described by Fick's laws of diffusion and can easily be quantified using the Cottrell equation ${ }^{12}$ for planar diffusion at macroelectrodes or the Shoup and Szabo approximation ${ }^{13}$ that additionally accounts for radial contributions at smaller electrodes $^{3,14}$ or in viscous solvents ${ }^{15,16}$.

In the majority of electrochemical studies, migration, the motion of charged species along an electric potential gradient, is omitted by the addition of an excess amount of 
electrochemically inert supporting electrolyte. The third process convection, however, is neither easily understood nor can it be omitted in many cases. While forced convection is negligible as long as external mechanical forces are excluded and natural convection due to temperature gradients ${ }^{17}$ can be avoided by tight thermostating of the entire electrochemical system, natural convection due to density gradients (buoyancy-driven convection) may again be an intrinsic feature of the electrochemical reaction itself. This is most evident in electrodeposition where the 'loss' of ions from the solution and hence a change in the density of the solution near the electrode are intuitively apparent, ${ }^{18,19}$ but it is equally true for solution-phase redox couples due to the typically large change of their solvation shells associated with the change of the species' charge upon gaining or losing an electron. ${ }^{20,21}$ Up until now, it has often been implied that this density gradient driven natural convection is only significant when high concentrations of redox-active species are concerned ${ }^{22,23}$ or when homogeneous chemical reactions are coupled to the electrochemical reaction ${ }^{22}$. Instead, spontaneous convection due to the chaotic motion of microscopic fluid elements has been suggested as another convective mode. Equations to quantify the effect of this flow on mass transport have been derived by Amatore et al. ${ }^{23}$ by analogy to turbulent fluid flows and have successfully been applied in a number of electrochemical studies. ${ }^{11,24-30}$ The driving force for such 'spontaneous' convection is uncertain and indeed the term may be slightly misleading in the sense that the origins of the effect are difficult to quantify in the absence of a clear understanding of the relevant driving forces. For this reason, we focus exclusively on density difference driven convection.

However, when macroelectrodes are concerned, significant density gradients across the electrode surface upon an electrochemical reaction cannot generally be avoided, making them more prone to density driven convection than (ultra-) microelectrodes. Here we investigate and quantify the conditions that allow for density driven natural convection to safely be 
excluded and those where not. For this purpose, in depth numerical simulations are performed for one of the most frequently studied redox couples, hexacyanoferrate(II) / hexacyanoferrate(III) $\quad\left(\left[\mathrm{Fe}(\mathrm{CN})_{6}\right]^{4-} /\left[\mathrm{Fe}(\mathrm{CN})_{6}\right]^{3-}\right)$. The effects of different electrode sizes, electrode orientations with respect to gravity and experimental timescales on the evolution of density gradient driven natural convection are analysed using numerical simulation. The resulting velocity profiles and the influence of natural convection on the diffusion layer established and on the currents observed during chronoamperometric oxidation of $\left[\mathrm{Fe}(\mathrm{CN})_{6}\right]^{4-}$ are quantified and discussed with respect to the currents obtained in the absence of convection. It is evident that for experimental timescales of tens of seconds and concentrations of sub $10 \mathrm{mM}$, natural convection may contribute significantly to the measured overall current. The flow fields established are presented for three exemplary electrode geometries.

\section{Theory}

In a chronoamperometric experiment of a 1-electron oxidation of the form,

$$
\mathrm{A} \rightleftarrows \mathrm{B}+\mathrm{e}^{-}
$$

the potential applied at the electrode is changed instantaneously from some initial potential, $E_{\mathrm{i}}$, which usually corresponds to zero current, to the potential, $E$, that provides a sufficiently high overpotential to overcome kinetic limitations, and thus enables studying of the mass transport of the electrochemical system. Throughout the duration $t$ of this mass transportcontrolled oxidation of A, all species A arriving at the electrode are immediately oxidized and the concentration of this species at the electrode surface is effectively zero. In this paper, we consider an ideally thermostated system that is not subjected to mechanical agitation and contains an excess amount of supporting electrolyte, ${ }^{31}$ so as to omit thermal convection, 
forced convection and migration and thus study mass transport due to diffusion and density gradient driven natural convection only.

We first consider diffusion-only systems in the absence of convection. The time-dependent current at a macroelectrode, governed by planar diffusion, are described by the Cottrell equation $^{12}$ :

$$
I_{\text {Cottrell }}=\frac{\mathrm{nF} A_{\mathrm{e}} \sqrt{D}}{\sqrt{\pi t}}[\mathrm{~A}]_{\mathrm{bulk}}
$$

where $\mathrm{n}$ is number of electrons transferred per ion/molecule $(\mathrm{n}=1$ for the hexacyanoferrate (II) / hexacyanoferrate (III) redox couple). $D$ is the diffusion coefficient and $[\mathrm{A}]_{\text {bulk }}$ is the bulk concentration of species $\mathrm{A}$ in the solution, $\mathrm{F}$ is the Faraday constant and $A_{\mathrm{e}}$ is the electrode area $\left(A_{\mathrm{e}}=\pi r_{\mathrm{e}}^{2}\right.$ for a disc electrode of radius $\left.r_{\mathrm{e}}\right)$.

The experimentally observed currents are often higher than predicted for pure planar diffusion. Two possible additional contributions to mass transport may account for that: radial diffusion and/or convection.

First, for relatively small 'macro' disk electrodes, radial diffusion contributes to the currents significantly when concentration gradients are not only established along the electrodenormal $z$-axis, but also along the $r$-axis (parallel to the electrode), for instance due to an insulating sheath surrounding the electrode (the axes are defined as in Fig. SI.I). The Shoup and Szabo equations ${ }^{13}$ (see eqn. 2.3 - 2.6) which take into account both the linear and radial diffusion components is therefore often more appropriate to analyse the data. ${ }^{32,33}$

$$
I_{\mathrm{SS}}=4 \mathrm{~F} D r_{\mathrm{e}}[\mathrm{A}]_{\mathrm{bulk}} f(\tau)
$$

where the dimensionless time $\tau$ is defined as

$$
\tau=4 D t r_{\mathrm{e}}^{-2}
$$

For short dimensionless times $(\tau<1), f(\tau)$ can be approximated as

$$
f(\tau)=\left(\frac{\pi}{4 \tau}\right)^{0.5}+\frac{\pi}{4}+0.094 \tau^{0.5}
$$


and for long times $(\tau>1)$

$$
f(\tau)=1+0.71835 \tau^{-0.5}+0.0562 \tau^{-1.5}-0.0064 \tau^{-2.5}
$$

The second possible contribution is by convection due to the local density gradients produced at the electrode when solutions of the redox species A and B have different densities (molar volumes). Due to the gravitational acceleration $\mathbf{g}$, these density gradients result in a bouyancy force that drives a convection and thus alters the mass transport and hence the concentration of species A and B in the electrochemical system.

Accounting for the conservation of mass, the resulting interdependence of diffusion and convection can be expressed by

$$
\frac{\partial c_{i}}{\partial t}+\nabla \cdot\left(c_{i} \boldsymbol{u}\right)-\nabla \cdot\left(D_{i} \nabla c_{i}\right)=0
$$

where $c_{i}$ is the concentration and $D_{i}$ is the diffusion coefficient of species $i, \boldsymbol{u}$ denotes the velocity vector and $t$ is the time. For a given fluid element, this expression balances the timedependent change in the concentration of species $i$ (caused by the electrochemical reaction, first term) to the convective transport (due to a velocity field $\boldsymbol{u}$, second term) and the diffusional transport (third term).

For an incompressible Newtonian fluid, the continuity equation which represents the conservation of mass is ${ }^{34}$

$$
\nabla \cdot \boldsymbol{u}=0
$$

and the conservation of momentum is described by the Navier-Stokes equation:

$$
\rho \frac{\partial \boldsymbol{u}}{\partial t}+\rho(\boldsymbol{u} \cdot \nabla) \boldsymbol{u}=-\nabla p+\mu \nabla^{2} \boldsymbol{u}+\mathbf{F}
$$

where $\rho$ is the density, $\boldsymbol{u}$ is the velocity vector, $p$ is the pressure, $\mu$ is the (constant) dynamic viscosity and $\mathbf{F}$ is the vector denoting additional volume forces.

In the study of natural convection due to density gradients, the volume force, $\mathbf{F}$, can be obtained from the Boussinesq approximation, provided that the density fluctuations in the 
system are small i.e. $\Delta \rho / \rho_{0} \ll 1$ and hence have no effect on the flow field except that they give rise to buoyant forces. ${ }^{35}$ The volume force is therefore set to

$$
\mathbf{F}=\rho_{0} \beta_{c}\left(c_{i}-c_{i}^{*}\right) \mathbf{g}
$$

where $\rho_{0}$ is the density of the fluid $\left(\mathrm{kg} \mathrm{m}^{-3}\right), \beta_{c}$ is the volume expansion coefficient defined as the change in molar volume from reactant to product $\left(\mathrm{m}^{3} \mathrm{~mol}^{-1}\right), c_{i}$ is the concentration and $c_{i}^{*}$ is the bulk concentration of species $i$. $\mathbf{g}$ is the gravity vector.

The numerical simulations are done for two different orientations of electrodes. In both cases, the electrode is horizontal, either facing downwards or facing upwards with respect to gravity as shown in Fig. 1a-b. A detailed description of the boundary conditions, numerical procedure and the parameters used in this work is given in the Supporting Information (SI), section SI.1.

\section{Results and Discussion}

As stated above, it has been suggested that natural convection due to density gradients is negligible in many systems at low concentrations of redox-active species and relatively short experimental timescales when micro or small macro electrodes are concerned. This assumption has major implications for a large number of analytical studies on electrode kinetics/electrocatalysis and so is worthwhile having a thorough analysis. ${ }^{36-39}$ In this work, we will show that the short timescale is not to be longer than 20s for one of the most frequently used redox systems even at sub $10 \mathrm{mM}$ concentrations.

In order to understand how natural convection affects mass transport to the electrode, we first look into the flow of the electrolyte solution and its effect on the diffusion layer/concentration gradient at the electrode. The concentration and velocity profiles for an electrode radius $\left(r_{\mathrm{e}}\right)$ of $1.5 \mathrm{~mm}$ are determined. The different effects of the electrode orientation with respect to gravity on the developed flow patterns and diffusion layers are then discussed. Afterwards, 
the influence of changing the electrode size on the patterns of the flow and the diffusion layer is analysed. Finally, the size- and time-dependent effects of non-planar diffusion and natural convection are rationalized and discussed for the different systems studied.

\subsection{Flow fields and concentration maps - downward-facing electrode $\left(r_{\mathrm{e}}=1.5 \mathrm{~mm}\right)$}

The development of the diffusion layer with time for an electrode of $1.5 \mathrm{~mm}$ radius facing in a horizontal downward orientation is shown in Fig. 1a. The thickness of the diffusion layer $(\delta)$ increases with time as more and more reactive species are being depleted close to the electrode surface. The values of $\delta$ are $284 \mu \mathrm{m}, 387 \mu \mathrm{m}$ and $445 \mu \mathrm{m}$ at $20 \mathrm{~s}, 40 \mathrm{~s}$ and $60 \mathrm{~s}$ respectively; slightly smaller than those resulted from the diffusion-only (convection-free, $\mathbf{u}=0, \mathbf{F}=0$ in eqn. 2.9, see SI.2) simulations which have $\delta$ values of $285 \mu \mathrm{m}, 403 \mu \mathrm{m}$ and $495 \mu \mathrm{m}$ at $20 \mathrm{~s}, 40 \mathrm{~s}$ and $60 \mathrm{~s}$ respectively. This diffusion layer remains relatively planar especially near the centre of the electrode surface. However, the radial diffusion at the edge of the electrode can only just be seen for this electrode size.

For the system studied, the hexacyanoferrate(III) product has a lower density than the hexacyanoferrate(II) reactant $\left(\beta_{c}=-5.9 \times 10^{-5} \mathrm{~m}^{3} \mathrm{~mol}^{-1} 40,41\right)$; therefore with the electrode facing downwards, the lighter product is formed and remains at the electrode surface because of buoyant force. This system can therefore be considered as a 'stable' configuration. The solution velocity is nevertheless non-zero except at $t=0$ s because a flow is driven by the difference in densities between the solution close to the inert sheath and the solution close to the active electrode surface.

At short times, for example at $t=10 \mathrm{~s}$, the maximum magnitude of the flow velocity is $2.7 \times 10^{-6} \mathrm{~m} \mathrm{~s}^{-1}$ which is relatively small and has a negligible effect on the diffusion layer thickness. At longer times, when the concentration layer becomes thicker, the volume force, $\mathbf{F}=\rho_{0} \beta_{c}\left(c_{i}-c_{i}^{*}\right) \mathbf{g}$, is enhanced because the portion of volume which has the concentration 
difference, $\left(c_{i}-c_{i}^{*}\right)$, increases, the force which drives the fluid flow then becomes bigger, and hence the magnitudes of the solution velocities increase with time. For $t=20 \mathrm{~s}, 40 \mathrm{~s}$ and $60 \mathrm{~s}$, the maximum magnitudes of the flow velocities are $4.8 \times 10^{-6} \mathrm{~m} \mathrm{~s}^{-1}, 7.5 \times 10^{-6} \mathrm{~m} \mathrm{~s}^{-1}$ and $8.7 \times 10^{-6} \mathrm{~m} \mathrm{~s}^{-1}$ respectively (see Fig. $1 \mathrm{~b}$ ). 
a)

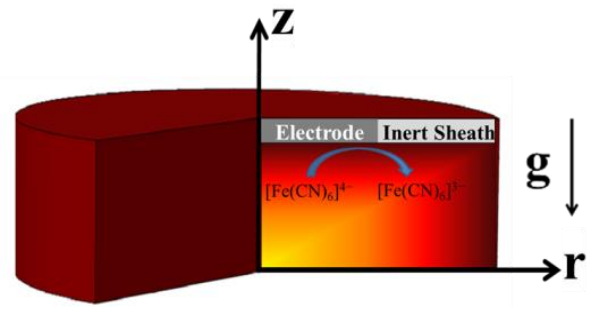

c)

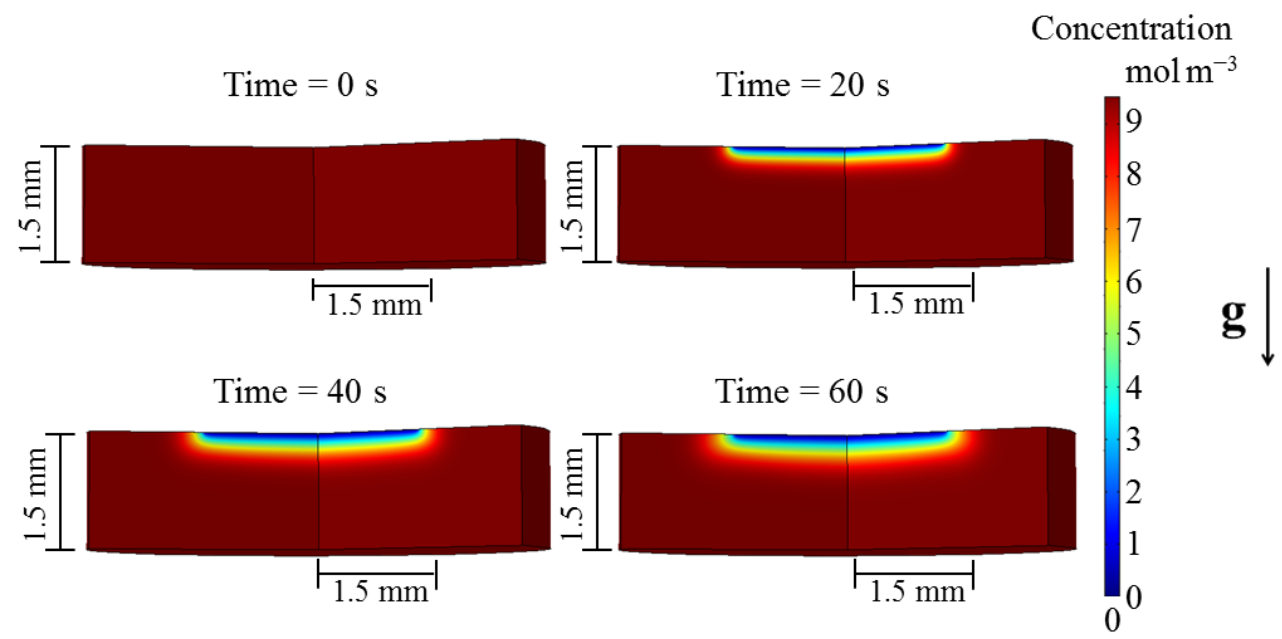

d)

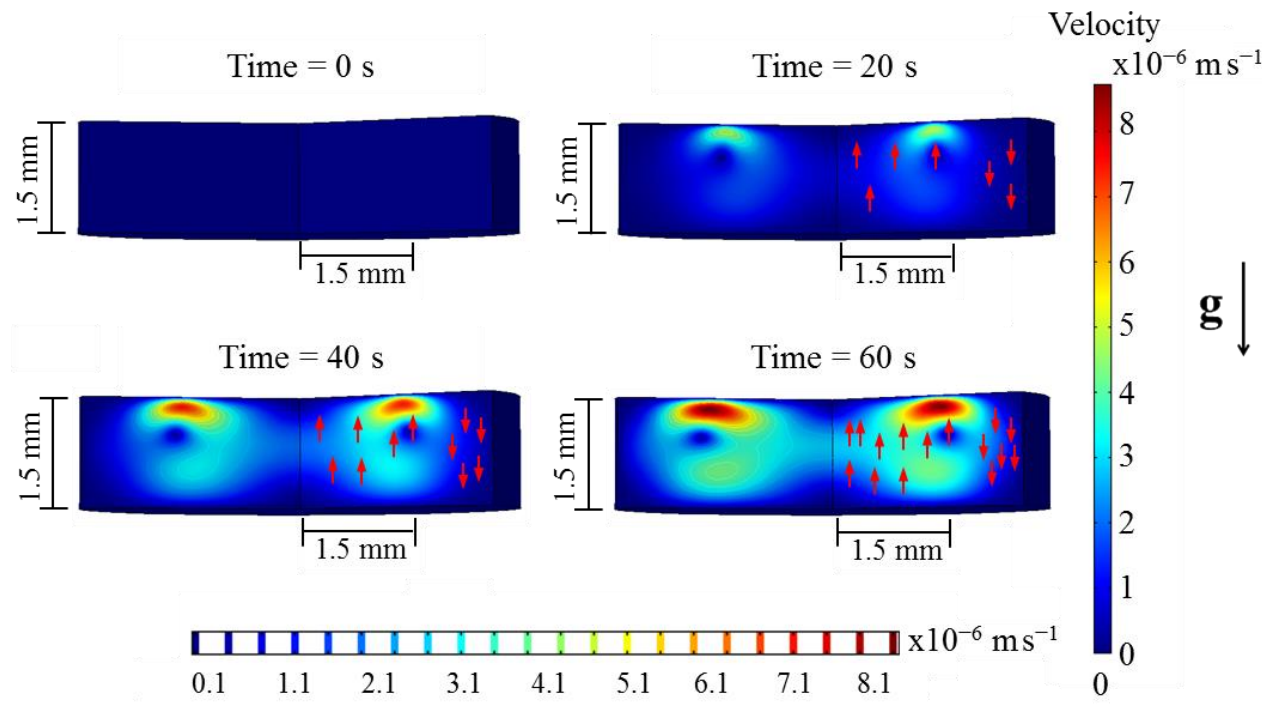

Figure 1: a) Schematic drawing of the electrotrochemical cell for electrode facing downwards and b) electrode facing upwards. c) Results of numerical simulations showing concentration and b) velocity profiles for the oxidation of $\left[\mathrm{Fe}(\mathrm{CN})_{6}\right]^{4-}$ at selected times $(0$, 20, 40 and 60s) for electrode $r_{\mathrm{e}}=1.5 \mathrm{~mm}$ facing downwards; the arrows indicate the direction of the flow, the magnitude of flow velocities is given by the colour representation (colour scale on the right-hand side) and contours (scale shown at the bottom); $c_{i}^{*}=9.5 \mathrm{~mol} \mathrm{~m}^{-3}, \mathrm{D}=7.53 \times 10^{-10} \mathrm{~m}^{2} \mathrm{~s}^{-1}{ }^{14}, \rho_{0}=1030 \mathrm{~kg} \mathrm{~m}^{-3} 42, \beta_{c}=-5.9 \times 10^{-5} \mathrm{~m}^{3} \mathrm{~mol}^{-1} 40,41$, $\mu=980 \times 10^{-6} \mathrm{~Pa} \mathrm{~s}^{42}$, for simulation details see SI section SI.1. 


\subsection{Flow fields and concentration maps - upward-facing electrode}

In contrast to the above, inverting the electrode orientation for otherwise identical parameters, a 'non-stable' configuration results as the lighter product is formed at the bottom of the electrochemical cell. Thus, the solution near the electrode is lighter than the bulk solution and an upward flow is driven by buoyant forces. In a closed system, this results in an opposite, downward flow near the edge of the cell.

The pattern of such flow and the maximum flow velocity, however, depend on the respective cell geometry (shape, height $z_{\max }$, width $r_{\max }$ and electrode size $r_{\mathrm{e}}$ ), due to the incompressibility of the fluid and the finite cell volume. Here we will discuss two different examples $\left(r_{\mathrm{e}}=1.5 \mathrm{~mm}\right.$ and $\left.r_{\mathrm{e}}=250 \mu \mathrm{m}\right)$ to emphasize this and to highlight the resulting impact on the diffusion layer in the vicinity of the electrode.

\section{Electrode facing upwards: $r_{\mathrm{e}}=1.5 \mathrm{~mm}$}

For the electrode of $1.5 \mathrm{~mm}$ radius, at short times $(t<20 \mathrm{~s})$, there is an upward flow directly above the electrode surface and a downward flow near the edge of the cell (i.e. above the insulation sheath); a simple flow pattern as explained above (see Fig. 2b, $t=20 \mathrm{~s}$ ). At longer times (see Fig. 2b, $t=40$ s and 60s), a more complex flow pattern is developed because a stream of fluid bounces off the solid surface and split into two. This complex flow pattern results in an inhomogeneous diffusion layer thickness across the electrode surface (see Fig 2a). The diffusion layer thickness is minimum at $r=0 \mathrm{~mm}$ with $\delta_{\min }$ of $286 \mu \mathrm{m}, 380 \mu \mathrm{m}$ and $270 \mu \mathrm{m}$ at $20 \mathrm{~s}, 40 \mathrm{~s}$ and $60 \mathrm{~s}$ respectively and maximum at $r=0.8 \mathrm{~mm}$ with $\delta_{\max }$ of $290 \mu \mathrm{m}$, $476 \mu \mathrm{m}$ and $946 \mu \mathrm{m}$ at $20 \mathrm{~s}, 40 \mathrm{~s}$ and 60 s respectively.

The maximum magnitudes of velocities are $\sim 50 \%$ higher than those for the electrode facing downwards at the same timescales, resulting in a stronger convective flow and more enhanced mass transport to the electrode surface. 
a)
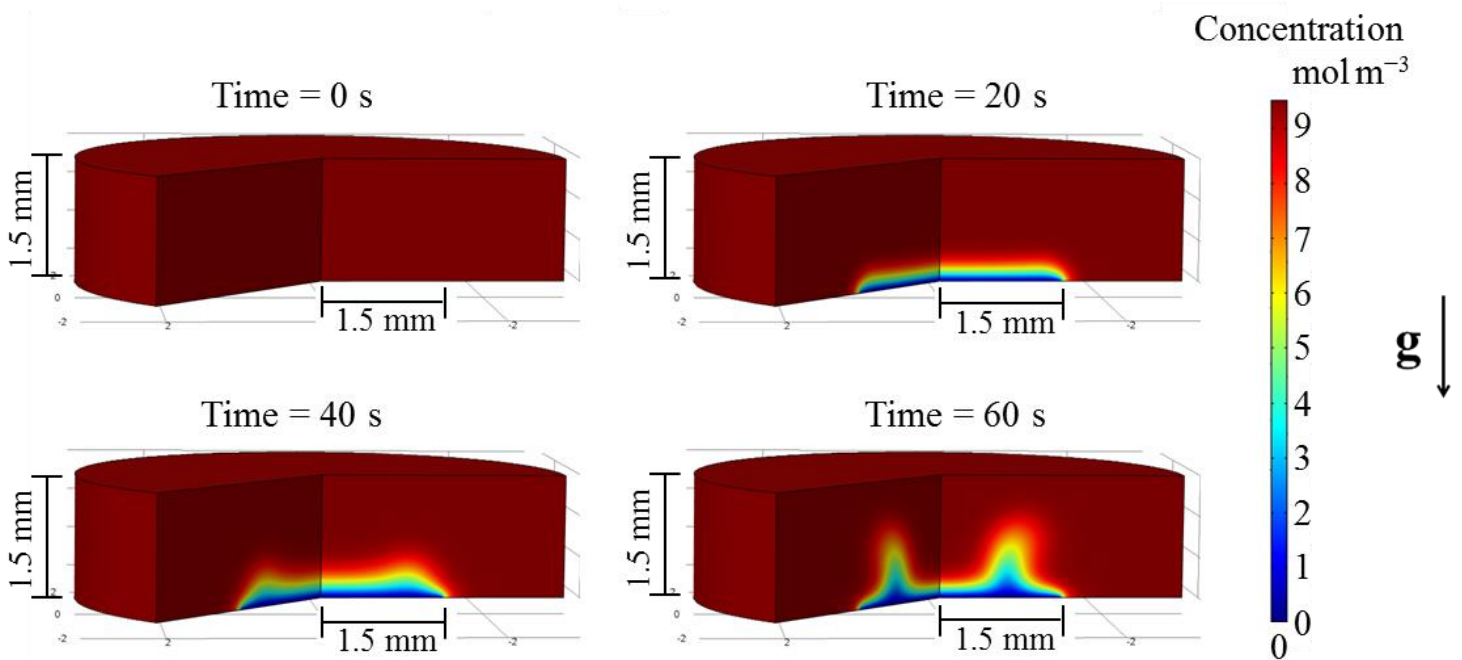

b)
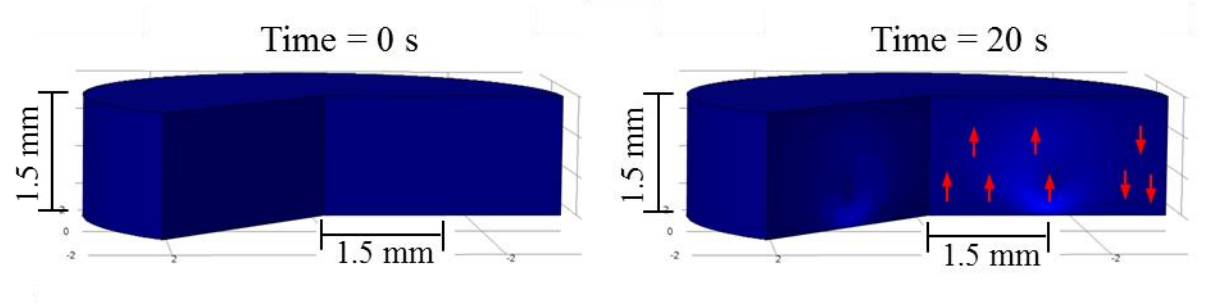

Velocity

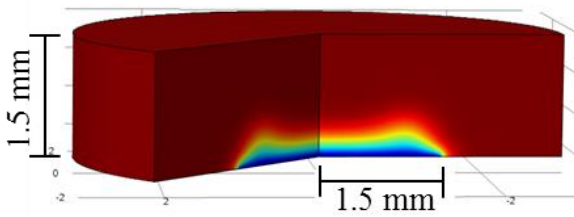

b)
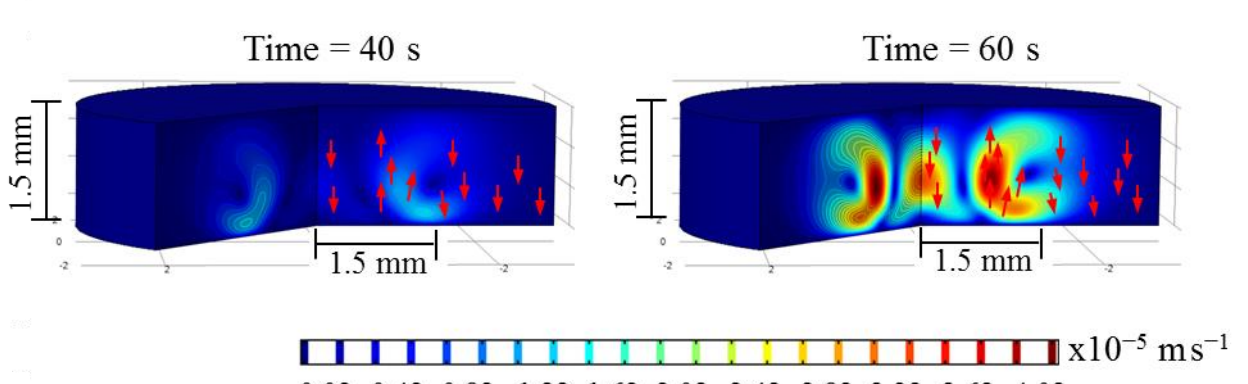

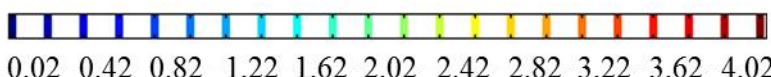

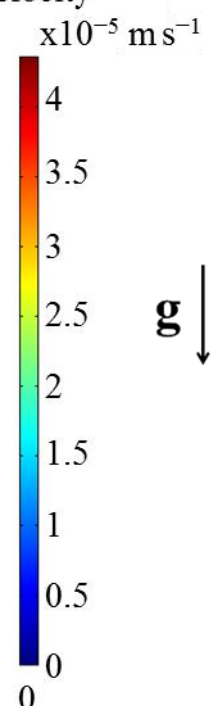

Figure 2: Results of numerical simulations showing a) concentration and b) velocity profiles for the oxidation of $\left[\mathrm{Fe}(\mathrm{CN})_{6}\right]^{4-}$ at selected times $(0 \mathrm{~s}, 20 \mathrm{~s}$, 40s and $60 \mathrm{~s})$ for electrode $r_{\mathrm{e}}=1.5 \mathrm{~mm}$ facing upwards; the arrows indicate the direction of the flow, the magnitude of flow velocities is given by the colour representation (colour scale on the right-hand side) and contours (scale shown at the bottom); $c_{i}^{*}=9.5 \mathrm{~mol} \mathrm{~m}^{-3}, \quad \mathrm{D}=7.53 \times 10^{-10} \mathrm{~m}^{2} \mathrm{~s}^{-1} \quad{ }^{14}$, $\rho_{0}=1030 \mathrm{~kg} \mathrm{~m}^{-3} 42, \beta_{c}=-5.9 \times 10^{-5} \mathrm{~m}^{3} \mathrm{~mol}^{-1} 40,41, \mu=980 \times 10^{-6} \mathrm{~Pa} \mathrm{~s}^{42}$, for simulation details see SI section SI.1. 


\subsection{Upward-facing electrode - electrode size effects $\left(r_{\mathrm{e}}=250 \mu \mathrm{m}\right)$}

Upon changing the electrode dimension, the flow fields and the diffusion layers established at the electrode are altered. When increasing the electrode radius from $1.5 \mathrm{~mm}$ to $4 \mathrm{~mm}$, no appreciable differences in the time evolution of macroscopic flow pattern and the diffusion layer shape were observed (see Supporting Information, section SI.3).

In contrast, for a microelectrode of $r_{\mathrm{e}}=250 \mu \mathrm{m}$, a different flow evolves and hence different concentration distribution at the electrode is observed, as illustrated for the 'non-stable' electrode orientation in Figure 3. At this microelectrode, an upward flow above the electrode surface and a downward flow near the edge of the cell (see Fig. 3b) are observed at all times (simulated up to $t=60 \mathrm{~s}$ ). The difference in the flow pattern from the bigger electrodes is because the Reynolds number $(\mathrm{Re})^{\S}$ changes significantly when changing the characteristic flow length, which in this case is the size of the electrode that creates the diffusion field.

The maximum magnitude of the flow velocity at $t=60 \mathrm{~s}$ is $\sim 3 \times 10^{-5} \mathrm{~m} \mathrm{~s}^{-1}$, slightly smaller but in the same order of magnitude as for the $r_{\mathrm{e}}=1.5 \mathrm{~mm}$ electrode. Natural convection due to density gradients is therefore significant even for microelectrodes at the timescales of tens of seconds.

The diffusion layer at short times $(t<20 \mathrm{~s})$ is not yet affected by convective flow, but instead of being planar, it is almost hemispherical in shape indicating the significant radial contribution. At $60 \mathrm{~s}$, the falling flow does not split into two as found for the $1.5 \mathrm{~mm}$ electrode because of the small electrode size relative to the cell diameter, and therefore the diffusion layer has a maximum thickness at the centre of the electrode surface (see Fig. 3a), while for the larger electrode a minimum diffusion layer thickness was observed at the same position. This clearly evidences the significant and diametrically different effect of natural convection

\footnotetext{
$\S$ The Reynolds number $(\operatorname{Re})$ is the ratio of inertial force to viscous force: $\operatorname{Re}=\rho \boldsymbol{u} L / \mu$, where $\rho$ is the density, $\boldsymbol{u}$ is the velocity, $L$ is the characteristic length and $\mu$ is the dynamic viscosity of the fluid.
} 
on these two electrode geometries (see also concentration profiles given in the Supporting Information, section SI.2).

a)
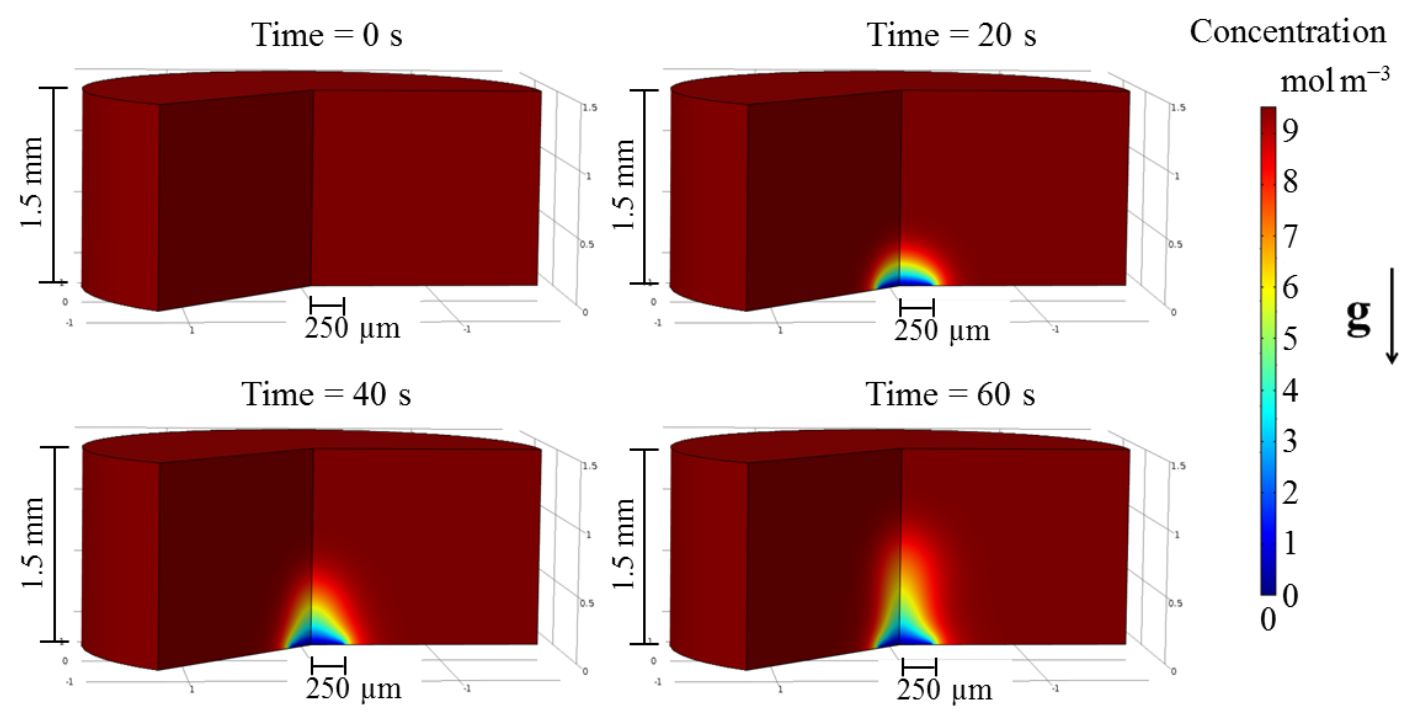

b)
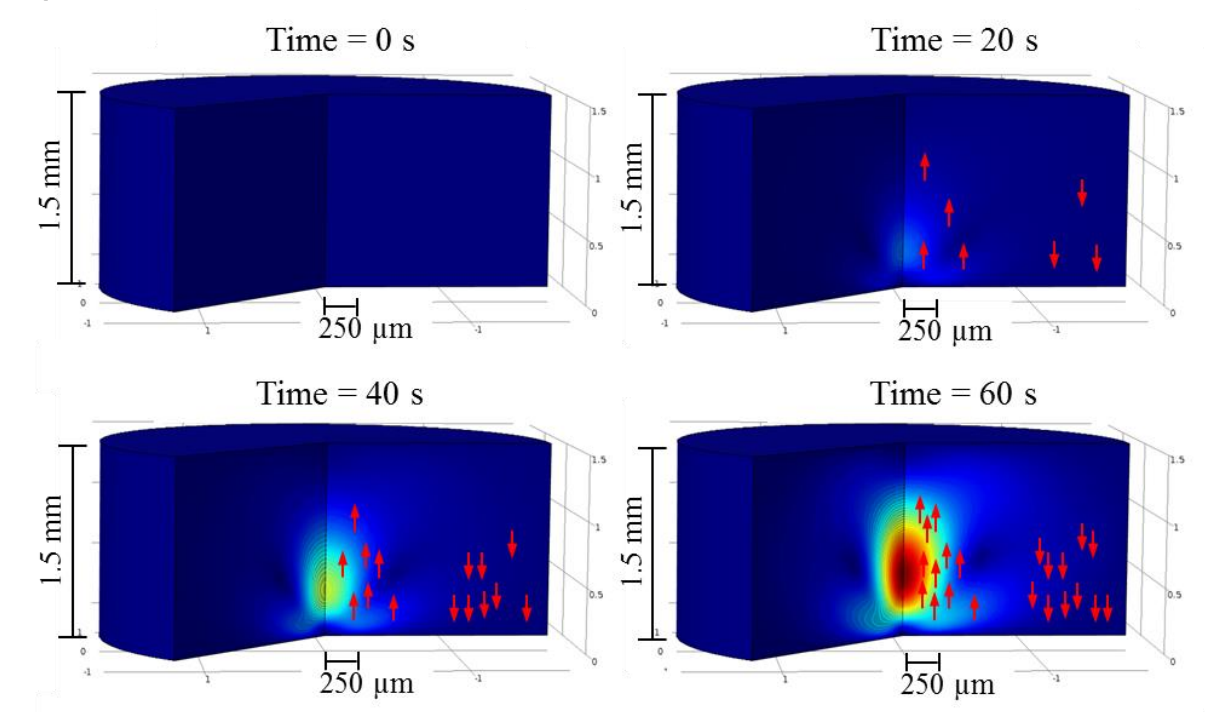

Velocity

Time $=40 \mathrm{~s}$
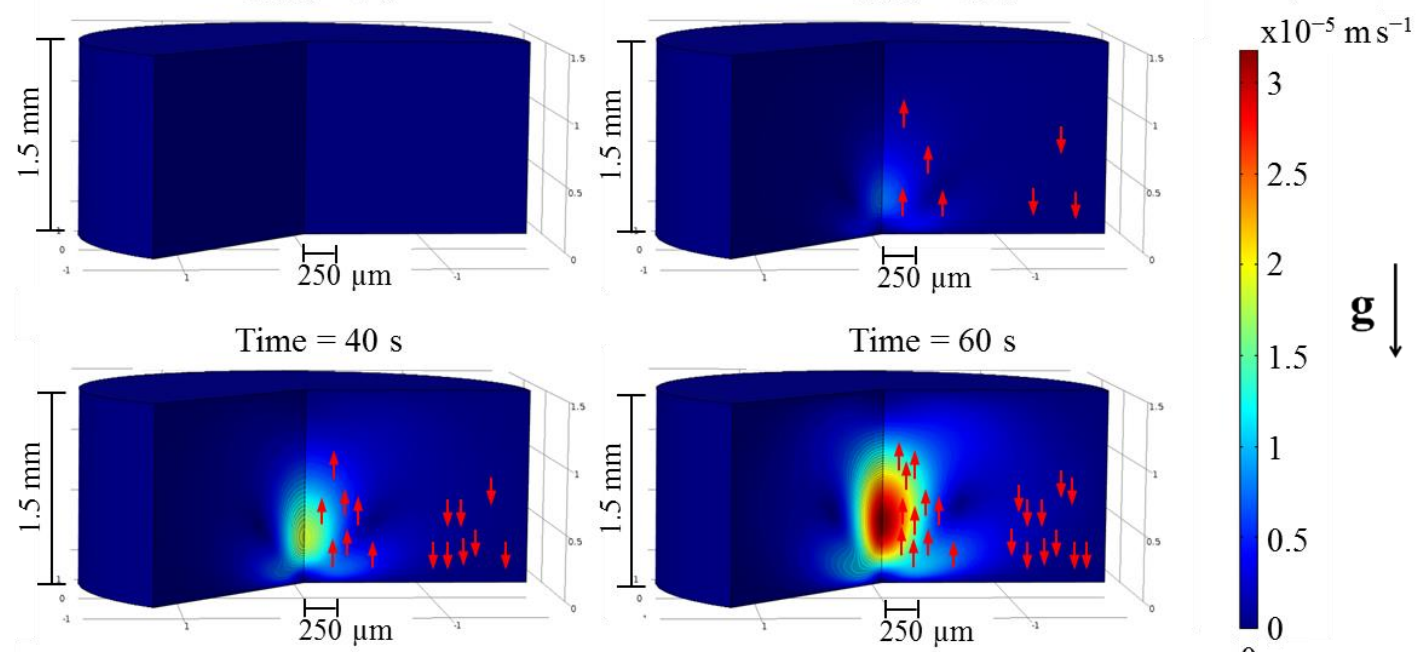

$\begin{array}{lllllllllll}0.1 & 0.4 & 0.7 & 1.0 & 1.3 & 1.6 & 1.9 & 2.2 & 2.5 & 2.8 & 3.1\end{array}$

Figure 3: Results of numerical simulations showing a) concentration and b) velocity profiles for the oxidation of $\left[\mathrm{Fe}(\mathrm{CN})_{6}\right]^{4-}$ at selected times $(0 \mathrm{~s}, 20 \mathrm{~s}, 40 \mathrm{~s}$ and $60 \mathrm{~s})$ for electrode $r_{\mathrm{e}}=250 \mu \mathrm{m}$ facing upwards; the arrows indicate the direction of the flow, the magnitude of flow velocities is given by the colour representation (colour scale on the right-hand side) and contours (scale shown at the bottom); $\quad c_{i}^{*}=9.5 \mathrm{~mol} \mathrm{~m}^{-3}, \quad \mathrm{D}=7.53 \times 10^{-10} \mathrm{~m}^{2} \mathrm{~s}^{-1}{ }^{14}$, $\rho_{0}=1030 \mathrm{~kg} \mathrm{~m}^{-3}{ }^{42}, \beta_{c}=-5.9 \times 10^{-5} \mathrm{~m}^{3} \mathrm{~mol}^{-1} 40,41, \mu=980 \times 10^{-6} \mathrm{~Pa} \mathrm{~s}^{42}$, for simulation details see SI section 1 . 


\subsection{Effects on Chronoamperometric Results}

As stated in the Introduction, the deviation of experimentally observed currents from the Cottrell equation in an unstirred/stagnant thermostated system may be caused by three possible contributions: radial diffusion, natural convection or spontaneous convection. First, we will show the results for diffusion-only simulations of different electrode sizes to demonstrate and quantify the radial contribution. Second, we will compare the results for simulations with and without the inclusion of the force driven by density gradients, then infer which of the two contributions dominates under which conditions. Spontaneous convection is not considered in this work, as its effects have been discussed in detail in numerous papers in the past. ${ }^{11,23-30}$

For the diffusion-only simulations of currents at electrodes of different sizes: $250 \mu \mathrm{m}, 1.5 \mathrm{~mm}$ and $4.0 \mathrm{~mm}$, all of which were often described as macroelectrodes and hence the radial contribution are often neglected..$^{25,43-45}$ However it has been previously reported by our group using cyclic voltammetry ${ }^{14}$ and is reinforced here using chronoamperometry that it is not completely true, and that the currents exceeding that predicted by the Cottrell equation are observed because of this radial diffusion, the so called edge effect.

The effect of this radial diffusion can be quantified by the current enhancement due to edge effect:

$$
\eta_{\text {edge }}=\frac{I(\mathbf{u}=0)-I(\text { Cottrell })}{I(\text { Cottrell })} \cdot 100 \%
$$

where $I$ (Cottrell) is calculated using eqn. 2.2 and $I(\mathbf{u}=0)$ is the total current obtained from a diffusion-only (convection-free) simulation using COMSOL as described in the Supporting Information, section SI.1.

The $\eta_{\text {edge }}$ results are given in Table 1 at $t=10 \mathrm{~s}, 20 \mathrm{~s}, 30 \mathrm{~s}, 40 \mathrm{~s}, 50 \mathrm{~s}$ and $60 \mathrm{~s}$ for all the three electrode sizes considered in this work. 
Current densities $(i)$, which are the currents per unit area of the electrode, should be identical for all electrode sizes if they follow the Cottrell linear diffusion. The larger current densities for smaller macroelectrodes therefore reflect the greater significance of radial diffusion in small electrode sizes (see Fig. 4).

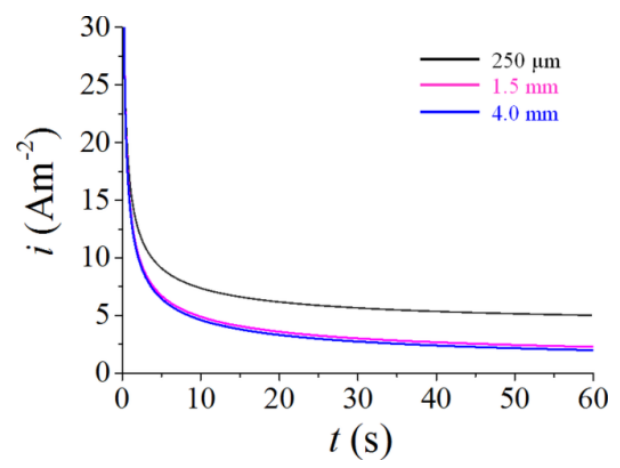

Figure 4: Current densities obtained from diffusion-only simulations of the $\left[\mathrm{Fe}(\mathrm{CN})_{6}\right]^{4-}$ oxidation for different sizes of electrodes and constant size of inert sheath $(1.5 \mathrm{~mm})$, $c_{i}^{*}=9.5 \mathrm{~mol} \mathrm{~m}{ }^{-3}, \mathrm{D}=7.53 \times 10^{-10} \mathrm{~m}^{2} \mathrm{~s}^{-114}$, for simulation details see $\mathrm{SI} .1$ )

Next, the focus of this paper is to evaluate the effect of natural convection generated by density gradients. Figure 5 shows the time-dependent currents obtained from numerical simulations of diffusion and convection employing the parameters given in the SI Tables SI.1 and SI.2.

The effect of natural convection is quantified by the current enhancement due to natural convection:

$$
\eta_{\mathrm{conv}}=\frac{I(\mathrm{conv})-I(\mathbf{u}=0)}{I(\mathbf{u}=0)} \cdot 100 \%
$$

where $I(\mathrm{conv})$ and $I(\mathbf{u}=0)$ are the time-dependent currents obtained from the simulations with and without the inclusion of density gradient driven natural convection respectively. (Note that we choose to use $\eta_{\text {conv }}$ as defined above rather than the Sherwood number $(\mathrm{Sh})^{* *}$

** The Sherwood number ( $\mathrm{Sh}$ ) is the ratio of convective to diffusive mass transfer: $\mathrm{Sh}=K L / D$, where $K$ is the mass transfer coefficient, $L$ is the characteristic length and $D$ is the diffusion coefficient. 
because we want to compare the significance of density gradient driven natural convection to the edge effect later on.)

The $\eta_{\text {conv }}$ results are given in Table 1 at $t=10 \mathrm{~s}, 20 \mathrm{~s}, 30 \mathrm{~s}, 40 \mathrm{~s}, 50 \mathrm{~s}$ and $60 \mathrm{~s}$ for all the three electrode sizes and for both electrode orientations. For all electrode sizes, the simulations which include the non-zero volume forces driven by the density gradients show higher currents than for the diffusion-only simulations. At short times $(t<20 \mathrm{~s})$, the effect of natural convection is small $(<1 \%)$ and therefore it is safe to assume that natural convection is negligible at these timescales since the difference in currents is smaller than typical experimental errors. This increase in currents is more significant at times longer than $30 \mathrm{~s}$ and in order to interpret the significance of natural convection to mass transport, we compare this to the edge effect using the $\eta_{\text {conv }} / \eta_{\text {edge }}$ ratio where $\eta_{\text {edge }}$ and $\eta_{\text {conv }}$ are defined as in eqn. 3.1 and 3.2 respectively.

Table 1: Summary of edge effects and natural convection effects for different electrode sizes in two orientations at different timescales

\begin{tabular}{|c|c|c|c|c|c|c|}
\hline$r_{\mathrm{e}}(\mathrm{mm}) \quad \mathrm{t}(\mathrm{s})$ & $10 \mathrm{~s}$ & $20 \mathrm{~s}$ & $30 \mathrm{~s}$ & $40 s$ & $50 \mathrm{~s}$ & $60 \mathrm{~s}$ \\
\hline \multicolumn{7}{|l|}{ Edge Effect } \\
\hline $250 \mu \mathrm{m}$ & $63 \%$ & $92 \%$ & $115 \%$ & $135 \%$ & $153 \%$ & $169 \%$ \\
\hline $1.5 \mathrm{~mm}$ & $9 \%$ & $13 \%$ & $17 \%$ & $19 \%$ & $22 \%$ & $24 \%$ \\
\hline $4 \mathrm{~mm}$ & $3 \%$ & $5 \%$ & $6 \%$ & $7 \%$ & $8 \%$ & $9 \%$ \\
\hline \multicolumn{7}{|l|}{ Natural Convection } \\
\hline \multicolumn{7}{|l|}{ electrode facing downwards } \\
\hline $250 \mu \mathrm{m}$ & - & - & $1 \%$ & $2 \%$ & $3 \%$ & $4 \%$ \\
\hline $1.5 \mathrm{~mm}$ & - & - & $1 \%$ & $2 \%$ & $3 \%$ & $4 \%$ \\
\hline $4 \mathrm{~mm}$ & - & - & - & $1 \%$ & $1 \%$ & $2 \%$ \\
\hline \multicolumn{7}{|l|}{ electrode facing upwards } \\
\hline $250 \mu \mathrm{m}$ & - & $1 \%$ & $3 \%$ & $7 \%$ & $12 \%$ & $17 \%$ \\
\hline $1.5 \mathrm{~mm}$ & - & $1 \%$ & $2 \%$ & $7 \%$ & $18 \%$ & $38 \%$ \\
\hline $4 \mathrm{~mm}$ & - & - & $1 \%$ & $3 \%$ & $7 \%$ & $17 \%$ \\
\hline
\end{tabular}

The plots of these $\eta_{\text {conv }} / \eta_{\text {edge }}$ ratios for different electrode sizes and different orientations at different timescales are shown in Fig. 6. In general, the edge effect is much more significant than natural convection; only in the unstable configuration utilising relatively large macroelectrodes that the effect of natural convection is comparable to the edge effect. This observation is in agreement with previous experimental work by Gao et al. ${ }^{22}$ who reported the 
effects of natural convection on cyclic voltammetry responses using ultra micro electrodes in solutions containing $10 \mathrm{mM}$ hexacyanoferrate (II) or hexacyanoferrate (III). They found a smaller influence of natural convection for reduced electrode sizes in the range of $6.4 \mu \mathrm{m} 25$ $\mu \mathrm{m}$ in radius, due to the relatively larger contribution of radial diffusion; that is larger $\eta_{\text {edge }}$. In the same article, the effects of electrode orientations on chronoamperometric currents were also discussed, but only for much higher concentrations $(0.5 \mathrm{M})$ of the redox active species.

a)

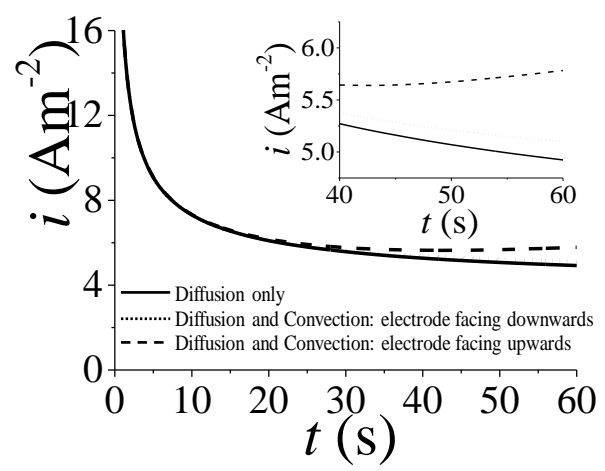

b)

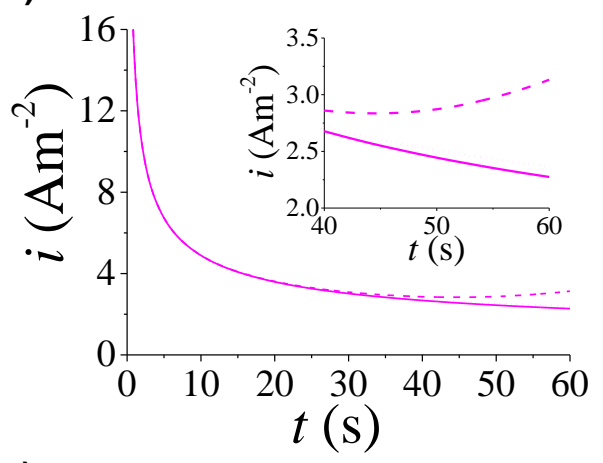

c)

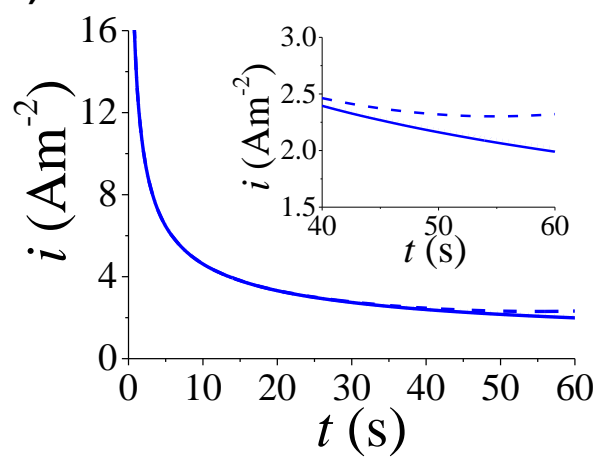

Figure 5: Current densities obtained from the simulations of the $\left[\mathrm{Fe}(\mathrm{CN})_{6}\right]^{4-}$ oxidation utilising electrodes of different radii a) $250 \mu \mathrm{m}$, b) $1.5 \mathrm{~mm}$ and c) $4.0 \mathrm{~mm}$ (see Fig.1 and Tables SI.1 and SI.2 for parameters); the continuous lines represent diffusion-only, dotted lines and dash lines represent diffusion+convection with electrodes facing downwards and upwards respectively. 
The increase in the currents observed for 'upwards facing' electrodes at ca. $50 \mathrm{~s}$ (see inlays Figs. 5a-c) marks the start of oscillating currents that were observed during the simulations at longer times and are in agreement with typical experimental observations of oscillating currents in electrochemical systems subject to natural convection. ${ }^{46}$

As both edge effect/radial diffusion and natural convection result in an increase in experimentally observed currents above those predicted by Cottrell, their respective significance may easily be misinterpreted. While the clear distinction of the two effects is difficult in the past, modern simulation tools and increasing computing power today enable us to clearly distinguish the two effects and quantify their respective significance for various experimental parameters such as electrode size, electrode orientation and experimental timescales. Moreover, aspects such as the electrochemical cell geometry and the redox couple studied have to be considered before attributing the current enhancement to either one of the two mass transport contributions.

a)

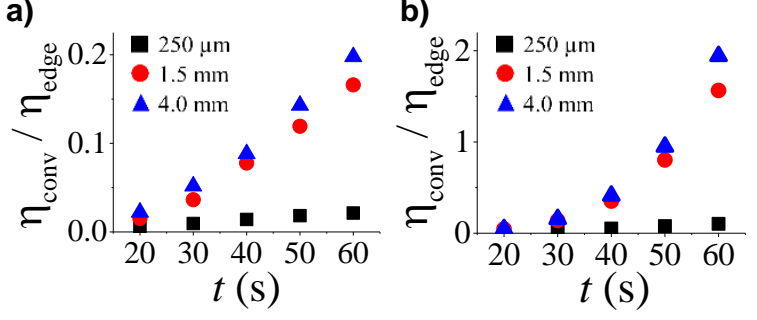

Figure 6: The ratio of edge effect to natural convection effect for a) electrode facing downwards and b) electrode facing upwards

\section{Conclusions}

In this paper, numerical simulation is used as an analytical tool to rationalize and quantify the significance of natural convection under different experimental conditions and at different timescales. Numerical simulation allows us to understand the behaviour (flow field) of the electrolyte solution subjected to natural convection and to obtain the shape and thickness of the diffusion layer; the information of which is valuable for many studies involving 
electrochemical reactions. Note that natural convection due to density gradients cannot be excluded from the systems with electrochemical reactions and cannot be neglected even at relatively low concentration of electroactive species, other than at short times (depending on the systems; $t<20 \mathrm{~s}$ for the hexacyanoferrate(II) / hexacyanoferrate(III) redox couple). The importance of electrode orientation with respect to gravity as a parameter to be considered when setting up an experiment is also emphasized.

Convective flow effectively reduces the thickness of the diffusion layer from that expected for the diffusion-only system and may result in the inhomogeneity of the diffusion layer which directly affects the results obtained from many electrochemical techniques; evident here for chronoamperometry. Similar effects are expected for voltammetric measurements at slow voltage scan rates $(\mathrm{d} E / \mathrm{d} t)$ where experimental timescales are sufficiently long for natural convection to develop.

As the inhomogeneity of the diffusion layers very clearly demonstrates, it is important to realize that analytical techniques which utilise electrochemical reactions to probe the surface reactivities or local surface effects in solution namely scanning electrochemical microscopy (SECM), generator-collector systems and indeed any macroelectrode or microelectrode array experiments have potential measurement errors. SECM, in particular, the substrate surface can be easily misunderstood as being electrochemically active when the probing electrode tip is at the position where the diffusion layer is relatively thinner than the nearby area; when actually it is just the result of natural convection. These misleading results may be minimized by choosing a redox couple which has a relatively small change in density upon an electrochemical reaction so as to minimize the convective flow.

The significance of natural convection driven by density gradients is further enhanced in systems with larger change in densities upon an electrochemical reaction, such as during electrodeposition or at higher concentration of redox species. 


\section{Acknowledgements}

KN acknowledges funding from the Royal Thai government under the Development and Promotion of Science and Technology Talents Project. KT was supported by a Marie Curie Intra European Fellowship under the FP 7 Framework Programme [no. 327706]. RGC acknowledges funding from the ERC Grant Agreement [no. 320403]. Supporting Information Available.

\section{References}

(1) Chen, W.; Wang, L.; Huang, X.; Wang, M. Anal. Chem. 2013, 85, 83.

(2) Dumitrescu, I.; Crooks, R. M. Proc. Natl. Acad. Sci. U. S. A. 2012, 109, 11493.

(3) Guo, S. X.; Zhang, J.; Elton, D. M.; Bond, A. M. Anal. Chem. 2004, 76, 166.

(4) Merkoci, A.; Pumera, M.; Llopis, X.; Perez, B.; del Valle, M.; Alegret, S. TrAC, Trends Anal. Chem. 2005, 24, 826.

(5) Pheeney, C. G.; Barton, J. K. J. Am. Chem. Soc. 2013, 135, 14944.

(6) Plumere, N.; Rudiger, O.; Oughli, A. A.; Williams, R.; Vivekananthan, J.;

Poller, S.; Schuhmann, W.; Lubitz, W. Nat. Chem. 2014, 6, 822.

(7) Sahore, V.; Fritsch, I. Anal. Chem. 2014, 86, 9405.

(8) Shao, Y. Y.; Wang, J.; Wu, H.; Liu, J.; Aksay, I. A.; Lin, Y. H. Electroanalysis 2010, 22, 1027.

(9) Stamenkovic, V. R.; Fowler, B.; Mun, B. S.; Wang, G.; Ross, P. N.; Lucas, C. A.; Markovic, N. M. Science 2007, 315, 493.

(10) Wang, J. Nucleic Acids Res. 2000, 28, 3011.

(11) Baltes, N.; Thouin, L.; Amatore, C.; Heinze, J. Angew. Chem., Int. Ed. 2004, 43,1431 .

(12) Cottrell, F. G. Z. Phys. Chem. 1902, 42, 385.

(13) Shoup, D.; Szabo, A. J. Electroanal. Chem. Interfacial Electrochem. 1982, 140, 237. 
(14) Ngamchuea, K.; Eloul, S.; Tschulik, K.; Compton, R. G. J. Solid State Electrochem. 2014, 18, 3251.

(15) Bentley, C. L.; Bond, A. M.; Hollenkamp, A. F.; Mahon, P. J.; Zhang, J. J. Phys. Chem. C 2014, 118, 29663.

(16) Bentley, C. L.; Bond, A. M.; Hollenkamp, A. F.; Mahon, P. J.; Zhang, J. J. Phys. Chem. C 2014, 118, 22439.

(17) Eckert, K.; Bestehorn, M.; Thess, A. J. Fluid Mech. 1998, 356, 155.

(18) Tschulik, K.; Cierpka, C.; Gebert, A.; Schultz, L.; Kahler, C. J.; Uhlemann, M. Anal. Chem. 2011, 83, 3275.

(19) Yang, X.; Mühlenhoff, S.; Nikrityuk, P. A.; Eckert, K. Eur. Phys. J.: Spec. Top. 2013, 220, 303.

(20) Hush, N. S. Trans. Faraday Soc. 1961, 57, 557.

(21) Waluyo, I.; Huang, C.; Nordlund, D.; Bergmann, U.; Weiss, T. M.; Pettersson, L. G.; Nilsson, A. J. Chem. Phys. 2011, 134, 064513.

(22) Gao, X.; Lee, J.; White, H. S. Anal. Chem. 1995, 67, 1541.

(23) Amatore, C.; Szunerits, S.; Thouin, L.; Warkocz, J.-S. J. Electroanal. Chem. 2001, 500, 62 .

(24) Amatore, C.; Pebay, C.; Thouin, L.; Wang, A.; Warkocz, J. S. Anal. Chem. 2010, 82, 6933.

(25) Amatore, C.; Szunerits, S.; Thouin, L.; Warkocz, J.-S. Electrochem. Commun. $2000,2,353$.

(26) Larchet, C.; Nouri, S.; Nikonenko, V. Desalination 2006, 200, 146.

(27) Amatore, C.; Pebay, C.; Thouin, L.; Wang, A. F. Electrochem. Commun. 2009, 11, 1269.

(28) Amatore, C.; Sella, C.; Thouin, L. J. Electroanal. Chem. 2006, 593, 194.

(29) Dolgikh, O.; Demeter, A. S.; Bastos, A. C.; Topa, V.; Deconinck, J. Electrochem. Commun. 2013, 37, 20.

(30) Demeter, A. S.; Dolgikh, O.; Bastos, A. C.; Deconinck, D.; Lamaka, S.; Topa, V.; Deconinck, J. Electrochim. Acta 2014, 127, 45.

(31) Dickinson, E. J. F.; Limon-Petersen, J. G.; Rees, N. V.; Compton, R. G. J. Phys. Chem. C 2009, 113, 11157.

(32) Xiong, L.; Aldous, L.; Henstridge, M. C.; Compton, R. G. Anal. Methods 2012, $4,371$. 
(33) Paddon, C. A.; Bhatti, F. L.; Donohoe, T. J.; Compton, R. G. J. Electroanal. Chem. 2006, 589, 187.

(34) Tritton, D. J. Physical Fluid Dynamics; Clarendon Press, 1988.

(35) Gray, D. D.; Giorgini, A. Int. J. Heat Mass Transfer 1976, 19, 545.

(36) Amatore, C. A.; Deakin, M. R.; Wightman, M. J. Electroanal. Chem. Interfacial Electrochem. 1986, 206, 23.

(37) Amatore, C.; Deakin, M. R.; Wightman, R. M. J. Electroanal. Chem. Interfacial Electrochem. 1987, 225, 49.

(38) Bond, A. M.; Oldham, K. B.; Zoski, C. G. J. Electroanal. Chem. Interfacial Electrochem. 1988, 245, 71.

(39) Bond, A. M. The Analyst 1994, 119, 1R.

(40) Selman, J. R.; Newman, J. J. Electrochem. Soc. 1971, 118, 1070.

(41) Volgin, V. M.; Davydov, A. D. Russ. J. Electrochem. 2011, 46, 1360.

(42) Haynes, W. M. CRC Handbook of Chemistry and Physics (Internet Version 2015) 95th ed.; CRC Press/Taylor and Francis, Boca Raton, FL.

(43) Nirmaier, H.-P.; Henze, G. Electroanalysis 1997, 9, 619.

(44) Nikolić, N. D.; Popov, K. I.; Ivanović, E. R.; Branković, G.; Stevanović, S. I.; Živković, P. M. J. Electroanal. Chem. 2015, 739, 137.

(45) Gomez-Mingot, M.; Montiel, V.; Banks, C. E.; Iniesta, J. The Analyst 2014, $139,1442$.

(46) Mühlenhoff, S.; Eckert, K.; Heinze, A.; Uhlemann, M. J. Electroanal. Chem. 2007, 611, 241. 


\section{For TOC Only}

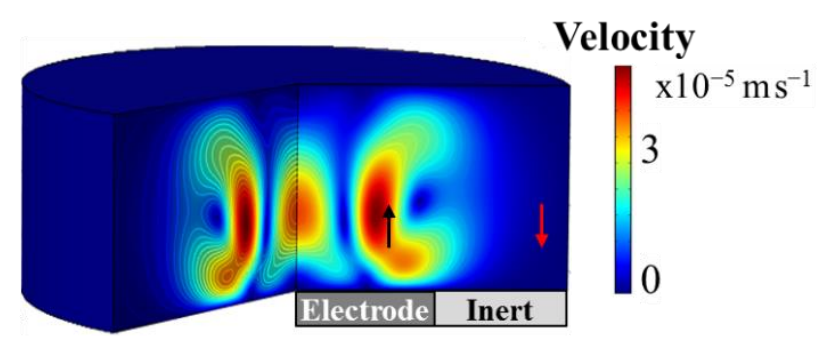

